\title{
Contract-Driven Implementation of Choreographies ${ }^{\star}$
}

\author{
Mario Bravetti, Ivan Lanese, and Gianluigi Zavattaro \\ Department of Computer Science, University of Bologna, Italy \\ \{bravetti, lanese, zavattar\}@cs.unibo.it
}

\begin{abstract}
Choreographies and Contracts are important concepts in Service Oriented Computing. Choreographies are the description of the behaviour of a service system from a global point of view, while contracts are the description of the externally observable message-passing behaviour of a given service. Exploiting some of our previous results about choreography projection and contract refinement, we show how to solve the problem of implementing a choreography via the composition of already available services that are retrieved according to their contracts.
\end{abstract}

\section{Introduction}

SENSORIA (Software Engineering for Service-Oriented Overlay Computers) 6 is a European project funded under the 6th Framework Programme as part of the Global Computing Initiative. The aim of SENSORIA is to develop a novel comprehensive approach to the engineering of software systems for service oriented computing where foundational theories, techniques and methods are fully integrated in a pragmatic software engineering approach.

Service Oriented Computing (SOC) is a paradigm for distributed computing based on services intended as autonomous and heterogeneous components that can be published and discovered via standard interface languages and publish/discovery protocols. Web Services is the most prominent service oriented technology: Web Services publish their interface expressed in WSDL, they are discovered through the UDDI protocol, and they are invoked using SOAP.

This paper addresses the problem of implementing service oriented systems, specified by means of high level languages called choreography languages in the SOC literature, by assembling already available services that can be automatically retrieved. The approach proposed in this paper in order to solve this problem is based on the assumption that services expose their behavioural interface expressed in terms of a contract, i.e. "the externally observable message-passing behaviour" 7].

More precisely, choreography languages are intended as notations for representing multi-party service compositions, that is, descriptions of the global behavior of service-based applications in which several services reciprocally communicate

\footnotetext{
* Research partially funded by EU Integrated Project Sensoria, contract n. 016004.

C. Kaklamanis and F. Nielson (Eds.): TGC 2009, LNCS 5474, pp. 118 2009.

(c) Springer-Verlag Berlin Heidelberg 2009
} 
in order to complete a predefined task. One of the most popular choreography languages has been developed by W3C and is called Web Services Choreography Description Language WS-CDL [13. In WS-CDL, the basic activities in a service choreography are interactions, that is, the atomic execution of a send and a receive operations performed by two communicating partners, called roles. For this reason, we say that WS-CDL follows an interaction-oriented approach.

When implementing an interaction-oriented choreography by assembling already available services, several problems are usually encountered. First of all, it is necessary to extract from the global specification the behaviour of each of the involved processes. After, one has to retrieve services exposing a behaviour which is compatible with the extracted processes. In previous papers, we have already considered these two problems in isolation.

In 9 we have considered a natural projection that, when applied to wellformed choreographies, extracts a set of processes corresponding to the expected behaviour of each role. More precisely, the main contribution of 9 is the syntactic characterization of well-formed choreographies and the formalization via variants of bisimilarity of the relation between a choreography and its projection.

In 244] we have developed a theory for contract refinement that allows one to replace a process in a given service system with a refined one preserving the correctness of the overall system. By correctness we mean that every computation of the system has one continuation leading to the successful completion of all combined services. One of the main contribution of 204] is the characterization of a property, called output persistence, that guarantees that the refinement of the processes in a system can be done independently (thus possibly in parallel). Output persistence holds when the decision to perform output operations is taken locally (i.e. it cannot be a guard in an external choice). This happens, for instance, in the most popular Web Services programming language WSBPEL [11. Technically, we obtain output persistence by changing the semantics of outputs, by splitting their execution in two parts: the execution of an internal action representing the decision to perform the output, and the subsequent synchronization with the corresponding receiver.

In this paper we show how to combine our previous results in order to solve the problem of checking whether an available service can play a given role in a given well-formed choreography. The simple intuition is that projection can be used to extract from the choreography the "maximal" process for the given role, then refinement can be used to check whether a service is a valid substitute for such "maximal" process. Nevertheless, in order to combine our previous results, we had to significantly revise both of them. As far as 9 is concerned, we have to modify the semantics of the choreography language following the approach in 24 by splitting in two parts the execution of the interactions. As we will discuss in the following, this modification requires the redefinition of the notion of well-formed choreography. As far as 224] is concerned, we have to define a new notion of contract refinement that considers, besides the correctness of a system, also the possible computations. In fact, we want to guarantee that the 
implementation of a choreography does not execute computations which are not admitted by the choreography.

The paper is structured as follows. In Section 2 we present the choreography and the contract languages. In Section 3 we discuss the projection function used to extract, from a choreography, the so-called "maximal" contract describing the expected behaviour of each role involved in the choreography. In Section 4 we present a theory for contract refinement suitable to verify whether a contract can be a valid substitute for one of the extracted "maximal" contract. Finally, in Section [5 we discuss how to combine the projection function and the contract refinement theory in order to define a conformance relation useful to check whether a service exposing a specific contract can implement a given role in a choreography. Section 6 reports some concluding remarks and a detailed discussion of our previous work about choreography projections and contract theories.

\section{Languages}

In this section we define two languages, one for expressing choreographies, inspired by WS-CDL, and one for expressing contract systems. In both the languages we assume a countable set $\mathcal{N}$ of operation names, ranged over by $o, o^{\prime}$, $\ldots$ and a countable set $\mathcal{R}$ of roles ranged over by $a, b, \ldots$

\subsection{Choreography Language}

The syntax of the choreography language is:

$$
\mathcal{I}::=o_{a \rightarrow b}|\mathbf{1}| \mathcal{I} ; \mathcal{I}^{\prime}\left|\mathcal{I} \| \mathcal{I}^{\prime}\right| \mathcal{I}+\mathcal{I}^{\prime}
$$

The basic construct is the interaction between two distinct roles $a$ and $b$ on operation $o$, denoted by $o_{a \rightarrow b}$. In addition there are the empty choreography 1, sequential and parallel composition of choreographies and nondeterministic choice between choreographies. For instance $\left(o_{a \rightarrow b} \| o_{a \rightarrow c}^{\prime}\right) ; o_{b \rightarrow c}^{\prime \prime}$ specifies that $o_{a \rightarrow b}$ and $o_{a \rightarrow c}^{\prime}$ can be performed in any order, and after both of them have been performed then $o_{b \rightarrow c}^{\prime \prime}$ can be executed. In the definition of the operational semantics we also consider the terminated choreography $\mathbf{0}$ and the ready interaction $\left\langle o_{a \rightarrow b}\right\rangle$.

In Table 1 we give an LTS semantics to choreographies. We use $\sigma$ to range over labels. Symmetric rules for parallel composition and choice have been omitted. We consider a delayed semantics: interactions are executed in two steps, an internal action first (rule TAU), followed by the real interaction (rule INTERACTION). We will use the same kind of delayed semantics also for contracts, and in that case it guarentees output persistency, a property that will be useful for contract refinement. The other rules are standard (see, e.g., [1).

We define the function $\operatorname{roles}(\mathcal{I})$ that given a choreography $\mathcal{I}$ computes the set of roles in it as:

$\operatorname{roles}\left(o_{a \rightarrow b}\right)=\{a, b\} \quad \operatorname{roles}(\mathbf{1})=\operatorname{roles}(\mathbf{0})=\emptyset$

$\operatorname{roles}\left(\mathcal{I} ; \mathcal{I}^{\prime}\right)=\operatorname{roles}\left(\mathcal{I} \| \mathcal{I}^{\prime}\right)=\operatorname{roles}\left(\mathcal{I}+\mathcal{I}^{\prime}\right)=\operatorname{roles}(\mathcal{I}) \cup \operatorname{roles}\left(\mathcal{I}^{\prime}\right)$ 
Table 1. Choreography semantics

$$
\begin{aligned}
& \text { (TAU) (INTERACTION) } \\
& o_{a \rightarrow b} \stackrel{\tau}{\longrightarrow}\left\langle o_{a \rightarrow b}\right\rangle \quad\left\langle o_{a \rightarrow b}\right\rangle \stackrel{o_{a \rightarrow b}}{\longrightarrow} \mathbf{1} \\
& \stackrel{(\text { END })}{\mathbf{1}} \stackrel{0}{\mathbf{0}} \quad \frac{\stackrel{(\text { SEQUENCE })}{\longrightarrow} \mathcal{I}^{\prime} \sigma \neq \sqrt{ }}{\mathcal{I} ; \mathcal{J} \stackrel{\sigma}{\longrightarrow} \mathcal{I}^{\prime} ; \mathcal{J}} \\
& \text { (Parallel) (Cholce) } \\
& \frac{\mathcal{I} \stackrel{\sigma}{\rightarrow} \mathcal{I}^{\prime} \sigma \neq \sqrt{ }}{\mathcal{I}\left\|\mathcal{J} \stackrel{\sigma}{\rightarrow} \mathcal{I}^{\prime}\right\| \mathcal{J}} \quad \frac{\mathcal{I} \stackrel{\sigma}{\rightarrow} \mathcal{I}^{\prime}}{\mathcal{I}+\mathcal{J} \stackrel{\sigma}{\rightarrow} \mathcal{I}^{\prime}} \\
& \text { (SEQ-END) }
\end{aligned}
$$

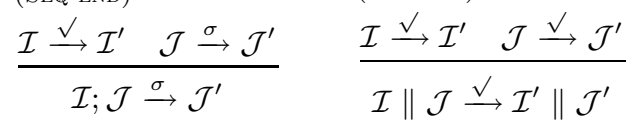

Table 2. Contract semantics

$$
\begin{aligned}
& \text { (In) (Out) (Local In) (Local Out) (Async-Out) (One) } \\
& \stackrel{\circ}{\rightarrow} \mathbf{1} \quad \bar{o} \stackrel{\tau}{\rightarrow}\langle o\rangle \quad o_{*} \stackrel{o_{*}}{\longrightarrow} \mathbf{1} \quad \bar{o}_{*} \stackrel{\bar{o}_{*}}{\longrightarrow} \mathbf{1} \quad\langle o\rangle \stackrel{\bar{o}}{\rightarrow} \mathbf{1} \quad \mathbf{1} \stackrel{\sqrt{ }}{\longrightarrow} \mathbf{0} \\
& \text { (SEQUENCE) (SEQ-END) } \\
& \frac{C \stackrel{\gamma}{\rightarrow} C^{\prime} \gamma \neq \sqrt{ }}{C ; D \stackrel{\gamma}{\rightarrow} C^{\prime} ; D} \quad \frac{C \stackrel{\sqrt{ }}{\longrightarrow} C^{\prime} \quad D \stackrel{\gamma}{\rightarrow} D^{\prime}}{C ; D \stackrel{\gamma}{\rightarrow} D^{\prime}} \\
& \text { (InNer Parallel) (Inner Par-end) (Local Synchro) } \\
& \frac{C \stackrel{\gamma}{\rightarrow} C^{\prime} \quad \gamma \neq \sqrt{ }}{C\left|D \stackrel{\gamma}{\rightarrow} C^{\prime}\right| D} \quad \frac{C \stackrel{\sqrt{ }}{\longrightarrow} C^{\prime} D \stackrel{\sqrt{ }}{\longrightarrow} D^{\prime}}{C\left|D \stackrel{\sqrt{ }}{\longrightarrow} C^{\prime}\right| D^{\prime}} \quad \frac{C \stackrel{o_{*}}{\longrightarrow} C^{\prime} D \stackrel{\bar{o}_{*}}{\rightarrow} D^{\prime}}{C\left|D \stackrel{\tau}{\rightarrow} C^{\prime}\right| D^{\prime}} \\
& \text { (Choice) (Local Res) } \\
& \frac{C \stackrel{\gamma}{\rightarrow} C^{\prime}}{C+D \stackrel{\gamma}{\rightarrow} C^{\prime}} \quad \frac{C \stackrel{\gamma}{\rightarrow} C^{\prime} \quad \gamma \notin\left\{o_{*}, \bar{o}_{*}\right\}}{C \backslash o_{*} \stackrel{\gamma}{\rightarrow} C^{\prime} \backslash o_{*}} \\
& \text { (INNER-Com) (INNER-T) } \\
& \frac{C \stackrel{\gamma}{\rightarrow} C^{\prime} \quad \gamma \in\{o, \bar{o}\}}{[C]_{a} \stackrel{\gamma: a}{\longrightarrow}\left[C^{\prime}\right]_{a}} \quad \frac{C \stackrel{\gamma}{\rightarrow} C^{\prime} \quad \gamma \in\{\tau, \sqrt{ }\}}{[C]_{a} \stackrel{\gamma}{\rightarrow}\left[C^{\prime}\right]_{a}} \\
& \text { (SYNCHRO) (EXT END) }
\end{aligned}
$$

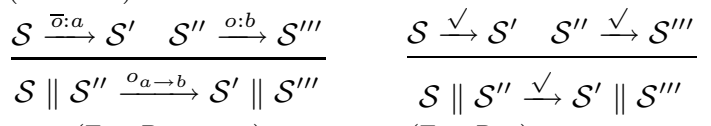

$$
\begin{aligned}
& \text { (Ext Parallel) (Ext Res) } \\
& \frac{\mathcal{S} \stackrel{\gamma}{\rightarrow} \mathcal{S}^{\prime} \quad \gamma \neq \sqrt{ }}{\mathcal{S}\left\|\mathcal{S}^{\prime \prime} \stackrel{\gamma}{\rightarrow} \mathcal{S}^{\prime}\right\| \mathcal{S}^{\prime \prime}} \quad \frac{\mathcal{S} \stackrel{\gamma}{\rightarrow} \mathcal{S}^{\prime} \quad \gamma \neq p}{\mathcal{S} \Downarrow p \stackrel{\gamma}{\rightarrow} \mathcal{S}^{\prime} \rrbracket p}
\end{aligned}
$$

\subsection{Contract Systems}

Contract systems, ranged over by $\mathcal{S}$, are compositions of located contracts, ranged over by $C$. For contract systems we consider also a set $\mathcal{N}_{\text {con }}=\left\{o_{*} \mid o \in\right.$ $\mathcal{N}\}$ for local operation names. We use $p$ for denoting either $o$ or $\bar{o}$. 


$$
\begin{aligned}
& \mathcal{S}::=[C]_{a}\left|\mathcal{S} \| \mathcal{S}^{\prime}\right| \mathcal{S} \Downarrow p \\
& C::=o|\bar{o}| o_{*}\left|\bar{o}_{*}\right| \mathbf{1}|\mathbf{0}| C ; C^{\prime}|C| C^{\prime}\left|C+C^{\prime}\right| C \backslash o_{*}
\end{aligned}
$$

Contract systems are compositions of roles. Each role includes a role name and a contract. We require role names to be unique. Roles can be composed in parallel, and actions can be restricted. The restriction $\ p$ is not a standard restriction in CCS-style, but it is asymmetric: $\ o$ forbids inputs on $o$, but lets outputs on the same operation go through, while $\Downarrow \bar{o}$ does the opposite.

Contracts include input $o$ and output $\bar{o}$ on a specific operation $o$, inputs $o_{*}$ and outputs $\bar{o}_{*}$ on local operations, the empty contract 1 and the terminated contract $\mathbf{0}$, sequential and parallel composition, nondeterministic choice and restriction of local names. The restriction of local names is a standard CCS-style restriction. The runtime syntax includes also messages $\langle o\rangle$.

We will use $\mathcal{S} \backslash\left\{p_{1}, \cdots, p_{n}\right\}$ as a shortcut for $\mathcal{S} \backslash p_{1} \cdots \Downarrow p_{n}$, and $\tau$ as shortcut for $\left(o_{*} \mid \bar{o}_{*}\right) \backslash o_{*}$.

The LTS for the contract semantics is in Table2 We use $\gamma$ to range over labels. Symmetric rules for parallel composition and choice have been omitted. Also for contract systems we consider a delayed semantics: an output $\bar{o}$ is executed in two steps, a $\tau$ action producing message $\langle o\rangle$ first (rule OUT), followed by the real communication (rule AsYNC-OUT). Inputs instead are not delayed, and the same holds for local operations. Notice that local operations cannot exit from roles (rules InNER-Com, InNER-T). The other rules are standard (see, e.g., [1]).

\section{From Choreographies to Contract Systems}

In this section we show how to relate choreographies and contract systems. In particular, given a choreography $\mathcal{I}$ we want to define a contract system $\mathcal{S}$ implementing it. The idea is to project the choregraphy on the different roles, and build the system $\mathcal{S}$ as parallel composition of the projections on the different roles.

The projection defined below is essentially an homomorphism. More complex projections can be defined, but they will be discussed in future work.

Definition 1 (Projection function). Given a choreography $\mathcal{I}$ and a role a, the projection $\operatorname{proj}(\mathcal{I}, a)$ of $\mathcal{I}$ on role $a$ is defined by structural induction on $\mathcal{I}$ :

$$
\begin{aligned}
\operatorname{proj}\left(o_{a \rightarrow b}, a\right) & =\bar{o} \\
\operatorname{proj}\left(o_{a \rightarrow b}, b\right) & =o \\
\operatorname{proj}\left(o_{a \rightarrow b}, c\right) & =\mathbf{1} \text { if } c \neq a, b \\
\operatorname{proj}\left(\left\langle o_{a \rightarrow b}\right\rangle, a\right) & =\langle o\rangle \\
\operatorname{proj}\left(\left\langle o_{a \rightarrow b}\right\rangle, b\right) & =o \\
\operatorname{proj}\left(\left\langle o_{a \rightarrow b}\right\rangle, c\right) & =\mathbf{1} \text { if } c \neq a, b \\
\operatorname{proj}(\mathbf{1}, a) & =\mathbf{1} \\
\operatorname{proj}(\mathbf{0}, a) & =\mathbf{0} \\
\operatorname{proj}\left(\mathcal{I} ; \mathcal{I}^{\prime}, a\right) & =\operatorname{proj}(\mathcal{I}, a) ; \operatorname{proj}\left(\mathcal{I}^{\prime}, a\right) \\
\operatorname{proj}\left(\mathcal{I} \| \mathcal{I}^{\prime}, a\right) & =\operatorname{proj}(\mathcal{I}, a) \mid \operatorname{proj}\left(\mathcal{I}^{\prime}, a\right) \\
\operatorname{proj}\left(\mathcal{I}+\mathcal{I}^{\prime}, a\right) & =\operatorname{proj}(\mathcal{I}, a)+\operatorname{proj}\left(\mathcal{I}^{\prime}, a\right)
\end{aligned}
$$

We denote with $\|_{i \in I} \mathcal{S}_{i}$ the parallel composition of contract systems $\mathcal{S}_{i}$ for each $i \in I$. 
Definition 2. Given a choreography $\mathcal{I}$, the associated contract system $\mathcal{S}$ is defined by:

$$
\operatorname{proj}(\mathcal{I})=\|_{a \in \operatorname{roles}(\mathcal{I})} \operatorname{proj}(\mathcal{I}, a)
$$

Notice that the projection never generates local actions nor restrictions, however these are useful for contract refinement.

The projection $\operatorname{proj}(\mathcal{I})$ of a given choreography $\mathcal{I}$ is a system that behaves according to the choreography $\mathcal{I}$. However this is guarenteed only for wellformed choreographies. Let us consider for instance the simple choreography $\mathcal{I}=o_{a \rightarrow b} ; o_{c \rightarrow d}^{\prime}$. Intuitively, interaction at $o$ should occur before the interaction at $o^{\prime}$. However, the projection of this choreography is the contract system $\mathcal{S}=[\bar{o} ; \mathbf{1}]_{a}\left\|[o ; \mathbf{1}]_{b}\right\|\left[\mathbf{1} ; \overline{o^{\prime}}\right]_{c} \|[\mathbf{1} ; o]_{d}$. Here we have the possible computation $\mathcal{S} \stackrel{\tau}{\rightarrow} \stackrel{o_{c \rightarrow d}^{\prime}}{\longrightarrow}[\bar{o} ; \mathbf{1}]_{a}\left\|[o ; \mathbf{1}]_{b}\right\|[\mathbf{1}]_{c} \|[\mathbf{1}]_{d}$, which is not possible for the starting choreography. We will see below that choreography $\mathcal{I}$ does not satisfy connectedness for sequence.

We now formalize the semantic correspondance between a choreography and a contract system using the well-known notion of strong bisimilarity [10. As a simple corollary we get also a corresponding trace equivalence [8]. We also show that contract systems obtained by projecting a choreography are correct, i.e. they do not deadlock.

The definition of strong bisimilarity and strong trace equivalence below relate choreographies and contract systems. Notice also that for contract systems we consider just reductions, i.e. transitions with labels $o_{a \rightarrow b}, \tau$ or $\sqrt{ }$, and forbid instead unmatched inputs or outputs.

Definition 3 (Strong bisimilarity). A strong bisimulation is a relation $R$ between choreographies and contract systems such that if $(\mathcal{I}, \mathcal{S}) \in R$ then:

- if $\mathcal{I} \stackrel{\alpha}{\rightarrow} \mathcal{I}^{\prime}$ then $\mathcal{S} \stackrel{\alpha}{\rightarrow} \mathcal{S}^{\prime}$ and $\left(\mathcal{I}^{\prime}, \mathcal{S}^{\prime}\right) \in R$;

- if $\mathcal{S} \stackrel{\gamma}{\rightarrow} \mathcal{S}^{\prime}$ with $\gamma$ of the form $o_{a \rightarrow b}, \tau$, or $\sqrt{ }$ then $\mathcal{I} \stackrel{\gamma}{\rightarrow} \mathcal{I}^{\prime}$ and $\left(\mathcal{I}^{\prime}, \mathcal{S}^{\prime}\right) \in R$.

Strong bisimilarity $\sim$ is the largest strong bisimulation.

Definition 4 (Strong trace equivalence). A trace of a choreography $\mathcal{I}_{1}$ is a sequence of labels $\alpha_{1}, \ldots, \alpha_{n}$ such that there is a sequence of choreography transitions $\mathcal{I}_{1} \stackrel{\alpha_{1}}{\longrightarrow} \ldots \stackrel{\alpha_{n}}{\longrightarrow} \mathcal{I}_{n+1}$.

A trace of a contract system $\mathcal{S}_{1}$ is a sequence of labels $\gamma_{1}, \ldots, \gamma_{n}$, where $\gamma_{i}$ is of the form $o_{a \rightarrow b}, \tau$, or $\sqrt{ }$ for each $i \in\{1, \ldots, n\}$, such that there is a sequence of contract system transitions $\mathcal{S}_{1} \stackrel{\gamma_{1}}{\longrightarrow} \ldots \stackrel{\gamma_{n}}{\longrightarrow} \mathcal{S}_{n+1}$

A choreography trace (resp. contract system trace) is complete when $\alpha_{n}=\sqrt{ }$ (resp. $\left.\gamma_{n}=\sqrt{ }\right)$.

A choreography $\mathcal{I}$ and a contract system $\mathcal{S}$ are strong trace equivalent iff they have the same set of complete traces.

We define now the notion of correct contract system.

Definition 5 (Correct contract system). A contract system $\mathcal{S}_{1}$ is correct, denoted $\mathcal{S}_{1} \downarrow$, if given a sequence of transitions $\mathcal{S}_{1} \stackrel{\gamma_{1}}{\longrightarrow} \ldots \stackrel{\gamma_{n}}{\longrightarrow} \mathcal{S}_{n+1}$ where $\gamma_{i}$ is 
of the form $o_{a \rightarrow b}, \tau$, or $\sqrt{ }$ for each $i \in\{1, \ldots, n\}$, there exists a continuation of the sequence $\mathcal{S}_{n+1} \stackrel{\gamma_{n+1}}{\longrightarrow} \ldots \stackrel{\sqrt{ }}{\longrightarrow} \mathcal{S}_{m}$ where $\gamma_{j}$ is of the form $o_{a \rightarrow b}, \tau$, or $\sqrt{ }$ for each $j \in\{n+1, \ldots, m-1\}$.

We want to ensure that a choreography $\mathcal{I}$ is bisimilar to its projection $\operatorname{proj}(\mathcal{I})$. As we have already seen, this is not always the case. We will describe below some well-formedness conditions that ensure this property. Since these conditions are syntactic, they can be easily checked by analyzing the starting choreography. We call them connectedness conditions. We have three kinds of connectedness conditions, one for sequential composition (connectedness for sequence), one for nondeterministic choice (unique point of choice), and one for operations used many times (causality-safety). Interestingly, no special condition is required for parallel composition. The connectedness conditions are formalized below.

We start by introducing a few auxiliary functions. Functions transI(•) and $\operatorname{transF}(\bullet)$ compute respectively the sets of initial and final interactions in a choreography:

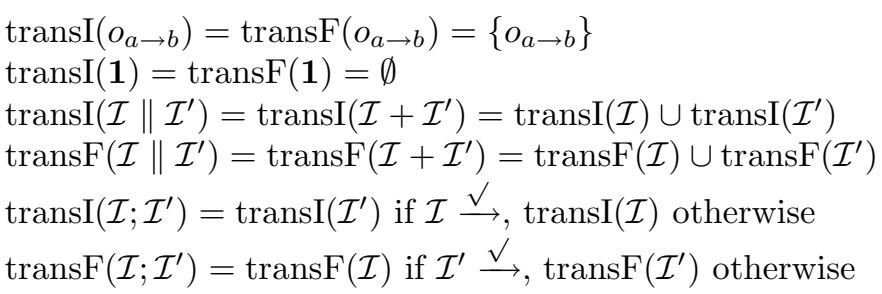

The definition of connectedness for sequence intuitively ensures that if two interactions have to be executed in sequence because of a sequential composition in the starting choreographjy, then there is a role $a$ which is involved in both the interactions. Thus a sequential composition will occur also in the projection on $a$, and the sequentiality constraint will be satisfied.

Definition 6 (Connectedness for sequence). A choreography $\mathcal{I}$ is connected for sequence if for each subterm $\mathcal{I} ; \mathcal{J}$ we have $\forall o_{a \rightarrow b} \in \operatorname{transF}(\mathcal{I}), \forall o_{c \rightarrow d}^{\prime} \in$ $\operatorname{transI}(\mathcal{J}),\{a, b\} \cap\{c, d\} \neq \emptyset$.

For choice two things are necessary: the choice has to be taken by a unique role, otherwise there will be the risk of inconsistent choices, and all the roles involved in the following computations should be informed. The first condition is ensured by requiring that there is just one role $a$ which is the sender of all the possible starting messages. The second condition requires that the roles in all the branches of the choice are the same. Otherwise a role which is not involved in one of the branches may proceed before knowing whether it has to participate on the chosen branch or not.

Definition 7 (Unique point of choice). A choreography $\mathcal{I}$ has unique points of choice if for each subterm $\mathcal{I}+\mathcal{J}$ we have that $\forall o_{a \rightarrow b} \in \operatorname{transI}(\mathcal{I}), \forall o_{c \rightarrow d}^{\prime} \in$ $\operatorname{transI}(\mathcal{J}), a=c$. Furthermore $\operatorname{roles}(\mathcal{I})=\operatorname{roles}(\mathcal{J})$

Notice that the condition above is stronger then the one corresponding to the synchronous semantics in [9], in fact this corresponds to the one for the 
asynchronous semantics. This is due to the fact that the delayed semantics performs choices during the $\tau$ step, exactly as happens for the asynchronous semantics.

The two conditions above are enough when each operation occurs just once in the choreography. However the same operation can be used more than once, provided that special care is taken to ensure that different occurrences do not interfere. This is formalized below, requiring a causality relationship between interactions using the same operation.

For defining the causality relation we need to index interactions inside a choreography, and we use distinct natural numbers to this end. Indexes are preserved by the projection, i.e. the input and the output obtained by projecting interaction $i$ have both index $i$. We call a contract input and a contract output with the same index matching events. We denote with $\bar{e}$ the event matching event $e$. An event is unmatched if it has no matching events. An annotated choreography is a choreography with indexes.

Definition 8 (Causality relation). Let us consider an annotated choreography $\mathcal{I}$. A causality relation $\leq$ is a partial order among events in the projection $\mathcal{S}$ of $\mathcal{I}$. We define $\leq$ as the minimum partial order satisfying:

sequentiality: for each $\mathcal{I} ; \mathcal{I}^{\prime}$, if $i$ is an interaction in $\mathcal{I}, j$ is an interaction in $\mathcal{I}^{\prime}$, and $e_{i}$ and $e_{j}$ are events in the same role then $e_{i} \leq e_{j}$; synchronization: for each $i, j$ if $e_{i} \leq e_{j}$ then $\bar{e}_{i} \leq_{s} e_{j}$.

Definition 9 (Causality-safety). A choreography is causality-safe iff for each pair of interactions $i$ and $j$ using the same operation, either $s_{i} \leq r_{j} \wedge r_{i} \leq s_{j}$ or $s_{j} \leq r_{i} \wedge r_{j} \leq s_{i}$.

In order to understand the need for causality-safety consider the following choreography: $o_{a \rightarrow b} \| o_{c \rightarrow d}$. Here the two interactions exploit the same operation $o$, but there are no causal dependencies between the events corresponding to the two interactions, i.e., the choreography is not causality-safe. In fact the projection has the trace $[\bar{o} \mid \mathbf{1}]_{a}\left\|[o \mid \mathbf{1}]_{b}\right\|[\mathbf{1} \mid \bar{o}]_{c}\left\|[\mathbf{1} \mid o]_{d} \stackrel{\tau}{\longrightarrow} \stackrel{o_{a \rightarrow d}}{\longrightarrow}[\mathbf{1} \mid \mathbf{1}]_{a}\right\|[o \mid \mathbf{1}]_{b}\left\|[\mathbf{1} \mid \bar{o}]_{c}\right\|[\mathbf{1} \mid \mathbf{1}]_{d}$, which is not allowed by the choreography.

We can now state the main result of this section.

Theorem 1. Let $\mathcal{I}$ be a choreography and $\mathcal{S}=\operatorname{proj}(\mathcal{I})$ be its projection. If $\mathcal{I}$ satisfies the connectedness conditions then $\mathcal{I} \sim \mathcal{S}$.

Proof. Similar to the proof for the synchronous case in [9].

Corollary 1. Let $\mathcal{I}$ be a choreography and $\mathcal{S}=\operatorname{proj}(\mathcal{I})$ be its projection. If $\mathcal{I}$ satisfies the connectedness conditions then:

- $\mathcal{I}$ and $\mathcal{S}$ are strong trace equivalent;

$-\mathcal{S}$ is a correct contract system.

Proof. The first part is trivial, for the second one note that a choreography never deadlocks, thus the result follows from the first part. 


\section{Contract Refinement}

When implementing a choreography using already available services, usually it is not possible to retrieve services that behave exactly according to the projected contracts. To solve this problem, we consider a notion of contract refinement (similar to the one introduced in 2]) which allows us to detect whether a contract can safely replace another one when implementing a given choreography.

The refined contract system in general will not be strong trace equivalent to the starting choreography. We will characterize the behavioral relation between the starting choreography and the refined contract system using the weaker notion of weak trace refinement.

Definition 10 (Weak trace refinement). Given a trace $\gamma_{1}, \ldots, \gamma_{n}$ of a contract system $\mathcal{S}$ or of a choreography $\mathcal{I}$, the corresponding weak trace is obtained by removing all the $\tau$ labels. A complete weak trace is a weak trace corresponding to a complete trace.

A contract system $\mathcal{S}^{\prime}$ is a weak trace refinement of a contract system $\mathcal{S}$ if the set of complete weak traces of $\mathcal{S}^{\prime}$ is included in the set of complete weak traces of $\mathcal{S}$.

Given a contract system, we will show that it is possible to refine one of its contracts adding inputs on names that are not used by the other contracts in the system. This is possible because we parameterize our notion of refinement on the input and output names used in the context in which the refinement is actually applied. To this aim, we need to introduce the notion of input and output sets for contracts and contract systems.

Definition 11 (Input and Output sets). Given the contract $C$, we define $I(C)$ (resp. $O(C)$ ) as the subset of $\mathcal{N}$ of the potential input (resp. ouput) actions of $C$. Formally, we define $I(C)$ as follows:

$$
\begin{aligned}
& I(\mathbf{0})=I(\mathbf{1})=I\left(o_{*}\right)=I\left(\bar{o}_{*}\right)=I(\bar{o})=\emptyset \quad I(o)=\{o\} \\
& I\left(C ; C^{\prime}\right)=I\left(C+C^{\prime}\right)=I\left(C \mid C^{\prime}\right)=I(C) \cup I\left(C^{\prime}\right) \quad I\left(C \backslash o_{*}\right)=I(C)
\end{aligned}
$$

and $O(C)$ as follows:

$$
\begin{array}{ll}
O(\mathbf{0})=O(\mathbf{1})=O\left(o_{*}\right)=O\left(\bar{o}_{*}\right)=O(o)=\emptyset & O(\bar{o})=\{o\} \\
O\left(C ; C^{\prime}\right)=O\left(C+C^{\prime}\right)=O\left(C \mid C^{\prime}\right)=O(C) \cup O\left(C^{\prime}\right) & O\left(C \backslash o_{*}\right)=O(C)
\end{array}
$$

Note that $o_{*}$ in $C \backslash o_{*}$ does not influence $I\left(C o_{*}\right)$ and $O\left(C \backslash o_{*}\right)$ because it contains only local names outside $\mathcal{N}$. Given the system $\mathcal{S}$, we define $I(\mathcal{S})(\operatorname{resp} . O(\mathcal{S})$ ) as the subset of $\mathcal{N}$ of the potential input (resp. ouput) actions of $\mathcal{S}$. Formally, we define $I(\mathcal{S})$ as follows:

$$
\begin{aligned}
& I\left([C]_{a}\right)=I(C) \quad I\left(\mathcal{S} \| \mathcal{S}^{\prime}\right)=I(\mathcal{S}) \cup I(\mathcal{S}) \\
& I(\mathcal{S} \Downarrow p)= \begin{cases}I(\mathcal{S})-\{\text { o } 3 & \text { if } p=o \\
I(\mathcal{S}) & \text { otherwise }\end{cases}
\end{aligned}
$$


and $O(P)$ as follows:

$$
\begin{aligned}
& O\left([C]_{a}\right)=O(C) \quad O\left(\mathcal{S} \| \mathcal{S}^{\prime}\right)=O(\mathcal{S}) \cup O(\mathcal{S}) \\
& O(\mathcal{S} \backslash p)= \begin{cases}O(\mathcal{S})-\{\text { o } & \text { if } p=\bar{o} \\
O(\mathcal{S}) & \text { otherwise }\end{cases}
\end{aligned}
$$

In order to define our refinement in the most general way, i.e. capturing the coarsest notion of refinement, we proceed as follows. We first formalize the features that a relation should have in order to be a satisfactory subcontract relation. After, we investigate whether the union of all subcontract relations is still a subcontract relation. If this is the case, we can define our refinement as the maximum among all subcontract relations. A subcontract relation is a pre-order (a symmetric and transitive relation) on contracts that guarantees that when contracts are replaced by subcontracts in a correct system, the refined system is still correct and no new computations are added. As indicated above, this relation is parameterized on the sets $I, O$ of the names on which the other contracts in the context can perform input and output actions, respectively.

Definition 12 (Subcontract pre-order family). A family $\left\{\leq_{I, O} \mid I, O \subseteq\right.$ $\mathcal{N}\}$ of pre-orders over contracts is a subcontract pre-order family if, for any $n \geq 1$, contracts $C_{1}, \ldots, C_{n}$ and $C_{1}^{\prime}, \ldots, C_{n}^{\prime}$, input and output sets of names $I_{1}, \ldots, I_{n} \subseteq \mathcal{N}$ and $O_{1}, \ldots, O_{n} \subseteq \mathcal{N}$, and role names $a_{1}, \ldots, a_{n} \in \mathcal{R}$ we have that if

$$
\begin{aligned}
& \left(\left[C_{1}\right]_{a_{1}} \backslash I_{1} \cup \bar{O}_{1}\|\ldots\|\left[C_{n}\right]_{a_{n}} \| I_{n} \cup \bar{O}_{n}\right) \downarrow \wedge \\
& \forall i . C_{i}^{\prime} \leq_{I_{i}^{\prime}, O_{i}^{\prime}} C_{i} \wedge\left(\bigcup_{j \neq i} I\left(C_{j}\right)-I_{j}\right)-O_{i} \subseteq I_{i}^{\prime} \wedge\left(\bigcup_{j \neq i} O\left(C_{j}\right)-O_{j}\right)-I_{i} \subseteq O_{i}^{\prime}
\end{aligned}
$$

then

$$
\begin{aligned}
- & \left(\left[C_{1}^{\prime}\right]_{a_{1}} \Downarrow I_{1} \cup \bar{O}_{1}\|\ldots\|\left[C_{n}^{\prime}\right]_{a_{n}} \| I_{n} \cup \bar{O}_{n}\right) \downarrow \text { and } \\
- & \left(\left[C_{1}^{\prime}\right]_{a_{1}} \Downarrow I_{1} \cup \bar{O}_{1}\|\ldots\|\left[C_{n}^{\prime}\right]_{n} \| I_{n} \cup \bar{O}_{n}\right) \text { is a weak trace refinement of } \\
& \left(\left[C_{1}\right]_{a_{1}} \Downarrow I_{1} \cup \bar{O}_{1}\|\ldots\|\left[C_{n}\right]_{a_{n}} \| I_{n} \cup \bar{O}_{n}\right) .
\end{aligned}
$$

Note that it could be the case that a refined system has strictly less complete traces than the initial system. Consider for instance the system:

$$
\left[\bar{o}+\overline{o^{\prime}}\right]_{a} \|\left[o+o^{\prime}\right]_{b}
$$

and a subcontract pre-order such that

$$
\bar{o} \leq_{\left\{o, o^{\prime}\right\}, \emptyset} \bar{o}+\overline{o^{\prime}}
$$

Replacing the first contract with its subcontract we obtain the new refined system

$$
[\bar{o}]_{a} \|\left[o+o^{\prime}\right]_{b}
$$

in which the synchronization on $o^{\prime}$ is no longer possible.

We now have to check whether the union of all subcontract relations is still a subcontract relation. In fact, in general, this is not the case. For instance, if we consider the standard non-delayed output semantics, in which there is no previous internal $\tau$ action, we could consider the following correct system:

$$
\left[o+\overline{o^{\prime}}\right]_{a} \|\left[\bar{o}+o^{\prime}\right]_{b}
$$


and the two following subcontract relations $\leq_{\mathcal{N}-\left\{o^{\prime \prime}\right\}, \mathcal{N}-\left\{o^{\prime \prime}\right\}}^{1}, \leq_{\mathcal{N}-\left\{o^{\prime \prime}\right\}, \mathcal{N}-\left\{o^{\prime \prime}\right\}}^{2}$ such that

$o+\overline{o^{\prime}}+o^{\prime \prime} ; o \leq_{\mathcal{N}-\left\{o^{\prime \prime}\right\}, \mathcal{N}-\left\{o^{\prime \prime}\right\}}^{1} O+\overline{o^{\prime}} \quad$ and $\bar{o}+o^{\prime}+\overline{o^{\prime \prime}} ; o \leq_{\mathcal{N}-\left\{o^{\prime \prime}\right\}, \mathcal{N}-\left\{o^{\prime \prime}\right\}} \bar{o}+o^{\prime}$

It is easy to see that the union of the two subcontract relations is not a subcontract. In fact, the system

$$
\left[o+\overline{o^{\prime}}+o^{\prime \prime} ; o\right]_{a} \|\left[\bar{o}+o^{\prime}+\overline{o^{\prime \prime}} ; o\right]_{b}
$$

is not correct because it deadlocks after synchronization on $o^{\prime \prime}$.

In order to prove that in our specific case the union of all subcontract preorders is still a subcontract, we proceed as follows. We first define a weaker notion of subcontract pre-order (called Singular subcontract pre-order) in which we refine one contract only, leaving the rest of the system unchanged. It is obvious that each subcontract pre-order is also a singular pre-order, and that the union of all singular subcontract pre-orders (that we call Input-Output subcontract relation) is still a singular subcontract. In this way we obtain a relation which includes all subcontract pre-orders. Finally, we prove our main result in Theorem 2 where we show that also the opposite holds, i.e., the Input-Output subcontract relation is a subcontract pre-order.

We now define the Singular subcontract pre-order that allows one to refine only one contract in a contract system. Without loss of generality, we assume that the rest of the system which is left unchanged is in the form $\left(\left[C_{1}\right]_{a_{1}}\|\ldots\|\left[C_{n}\right]_{a_{n}}\right) \Downarrow I \cup \bar{O}$. We refer to systems in this particular form with $\mathcal{P}_{\text {conpar. }}$ We use $\mathcal{P}_{\text {conpar, }, O}$ to denote the set of systems $\mathcal{S}$ in $\mathcal{P}_{\text {conpar }}$ such that $I(\mathcal{S}) \subseteq I$ and $O(\mathcal{S}) \subseteq O$.

Definition 13 (Singular subcontract pre-order family). A family of preorders $\left\{\leq_{I, O} \mid I, O \subseteq \mathcal{N}\right\}$ is a singular subcontract pre-order family if, for any contracts $C, C^{\prime}$, role $a$, and $\mathcal{S} \in \mathcal{P}_{\text {conpar }}$ such that a does not appear in $\mathcal{S}$, we have that if $\left([C]_{a} \| \mathcal{S}\right) \downarrow \wedge C^{\prime} \leq_{I, O} C \wedge I(\mathcal{S}) \subseteq I \wedge O(\mathcal{S}) \subseteq O$ then

$-\left(\left[C^{\prime}\right]_{a} \| \mathcal{S}\right) \downarrow$ and

- $\left(\left[C^{\prime}\right]_{a} \| \mathcal{S}\right)$ is a weak trace refinement of $\left([C]_{a} \| \mathcal{S}\right)$.

As a pre-order is a symmetric relation, we have that singular refinement is admitted also by the usual subcontract relation simply replacing the other contracts with themselves. Based on this idea, it is possible to prove the following results.

Proposition 1. If a family of pre-orders $\left\{\leq_{I, O} \mid I, O \subseteq \mathcal{N}\right\}$ is a subcontract pre-order family then it is also a singular subcontract pre-order family.

Proof. Similar to the proof of the Proposition 3.1 in [4].

The proof of this proposition is similar to the one that can be found in [4]. We now define the union of all singular subcontract pre-orders.

Definition 14 (Input-Output Subcontract relation). A contract $C^{\prime}$ is a subcontract of a contract $C$ with respect to a set of input channel names $I \subseteq \mathcal{N}$ 
and output channel names $O \subseteq \mathcal{N}$, denoted $C^{\prime} \preceq_{I, O} C$, if for every role $a$ and every $\mathcal{S} \in \mathcal{P}_{\text {conpar, }, I, O}$ such that a does not appear in $\mathcal{S}$ we have that $\left([C]_{a} \| \mathcal{S}\right) \downarrow$ implies

$-\left(\left[C^{\prime}\right]_{a} \| \mathcal{S}\right) \downarrow$ and

- $\left(\left[C^{\prime}\right]_{a} \| \mathcal{S}\right)$ is a weak trace refinement of $\left([C]_{a} \| \mathcal{S}\right)$.

As a simple corollary of the Proposition 1 1 we have the following fact.

Corollary 2. Given a subcontract pre-order family $\left\{\leq_{I, O} \mid I, O \subseteq \mathcal{N}\right\}$, we have that it is included in $\left\{\preceq_{I, O} \mid I, O \subseteq \mathcal{N}\right\}$, that is

$$
C^{\prime} \leq_{I, O} C \Rightarrow C^{\prime} \preceq_{I, O} C
$$

We are now ready to prove our main result, i.e., that the input-output subcontract relation is also a subcontract pre-order. Combining this result with the above corollary, we have that the Input-Output subcontract relation is the maximum among all subcontract pre-orders.

Theorem 2. The family of pre-orders $\left\{\preceq_{I, O} \mid I, O \subseteq \mathcal{N}\right\}$ is a subcontract pre-order family.

Proof. Similar to the proof of the Theorem 3.2 in [4]. In fact, the additional requirement we have in this paper, i.e., that the set of complete traces of the refined system in included in the set of complete traces of the initial system, does not influence the proof of this result.

The remainder of this section is dedicated to the presentation of an actual procedure that can be used to check whether $C$ is a subcontract of $C^{\prime}$. In fact, the Definition 14 is not helpful in this perspective as it contains universal quantifications on every role $a$ and every system $\mathcal{S}$. We first observe that the knowledge about the input actions of the other contracts in the context is not relevant.

Proposition 2. Let $C$ be a contract, $O \subseteq \mathcal{N}$ be a set of output names and $I, I^{\prime} \subseteq \mathcal{N}$ be two sets of input names such that $O(C) \subseteq I, I^{\prime}$. We have that for every contract $C^{\prime}$,

$$
C^{\prime} \preceq_{I, O} C \quad \Longleftrightarrow \quad C^{\prime} \preceq_{I^{\prime}, O} C
$$

Proof. Similar to the proof of the Proposition 3.5 in [4].

This last proposition allows us to consider a simplified relation $C \preceq_{O} C^{\prime}$, that we call subcontract relation, in which only the knowledge about the output alphabet is relevant.

Definition 15 (Subcontract relation). A contract $C^{\prime}$ is a subcontract of a contract $C$ with respect to a set of output channel names $O \subseteq \mathcal{N}$, denoted $C^{\prime} \preceq_{O}$ $C$, if for every role a and for every $\mathcal{S} \in \mathcal{P}_{\text {conpar, }, \mathcal{N}, O}$ such that a does not appear in $\mathcal{S}$ we have that $\left([C]_{a} \| \mathcal{S}\right) \downarrow$ implies

$-\left(\left[C^{\prime}\right]_{a} \| \mathcal{S}\right) \downarrow$ and

- $\left(\left[C^{\prime}\right]_{a} \| \mathcal{S}\right)$ is a weak trace refinement of $\left([C]_{a} \| \mathcal{S}\right)$. 
We are now ready to present the description of the actual procedure that we can use to prove whether $C^{\prime} \preceq_{O} C$. The idea, discussed in [4, is to resort to the theory of should-testing [12; we define a transformation on $C$ and $C^{\prime}$, parameterized on the set $O$, such that if the transformed terms are in shouldtesting pre-order we can conclude that $C^{\prime} \preceq_{O} C$. The algorithm to check whether two terms are in should-testing pre-order is presented in [12.

In the following we denote with $\preceq_{\text {test }}$ the should-testing pre-order as defined in [12. The transformation that we apply on contracts returns terms of the language in [12]; this is necessary in order to be able to apply the algorithm presented in that paper. Namely, given a system $\mathcal{S}$ we define the transformation $\mathcal{N} \mathcal{F}\left({ }_{-}\right)$that returns a term corresponding to the process algebra in [12].

As we do not consider infinite behaviors, the labeled transition system of a system $\mathcal{S}$ is a finite tree with $\mathcal{S}$ as root. $\mathcal{N} F(\mathcal{S})$ is defined inductively on the height of the tree corresponding to the labeled transition system of $\mathcal{S}$ as follows:

$$
\mathcal{N} F(\mathcal{S})=\sum_{\left(\gamma, \mathcal{S}^{\prime}\right): \mathcal{S} \stackrel{\gamma}{\longrightarrow} \mathcal{S}^{\prime}} \gamma ; \mathcal{N} F\left(\mathcal{S}^{\prime}\right)
$$

where we assume empty sums to be equal to $\mathbf{0}$, i.e. if there are no outgoing transitions from $\mathcal{S}$, we have $\mathcal{N} \mathcal{F}(\mathcal{S})=\mathbf{0}$.

Theorem 3. Let $C, C^{\prime}$ be two contracts, $O \subseteq \mathcal{N}$ be a set of output names, and a be a role. We have

$$
\mathcal{N} \mathcal{F}\left(\left[C^{\prime}\right]_{a} \|(\mathcal{N}-O)\right) \preceq_{\text {test }} \mathcal{N} \mathcal{F}\left([C]_{a} \rrbracket(\mathcal{N}-O)\right) \quad \Rightarrow \quad C^{\prime} \preceq_{O} C
$$

Proof. We need to prove that if $\mathcal{N} \mathcal{F}\left(\left[C^{\prime}\right]_{a} \rrbracket(\mathcal{N}-O)\right) \preceq$ test $\mathcal{N} \mathcal{F}\left([C]_{a} \rrbracket(\mathcal{N}-O)\right)$ then we can replace $C$ with $C^{\prime}$ in any correct system (i) preserving correctness and (ii) without adding any additional complete trace. Result (i) is proved in [4] (see Theorem 3.3). Result (ii) follows from the fact that the should-testing preorder implies the trace refinement and it is a pre-congruence with respect to the parallel composition operator (see [12]).

\section{Conformance}

In this section we finally formalize the notion of conformance of one contract with a given role in a given choreography. As a first attempt, we could say that a contract $C$ is conformant to a role $a$ in a choreography $\mathcal{I}$ if services exposing the contract $C$ can be used in an implementation of $\mathcal{I}$ to play the role a. Unfortunately, we have that a conformance relation defined in this way is not satisfactory even if we restrict to well-formed choreographies. Consider, for instance, the following choreography:

$$
o_{a \rightarrow b} \| o_{a \rightarrow b}^{\prime}
$$

and the two following implementations:

$$
\left[\bar{o} \mid \overline{o^{\prime}}\right]_{a} \|\left[\tau ; o ; o^{\prime}+\tau ; o^{\prime} ; o\right]_{b} \quad \text { and } \quad\left[\bar{o} ; \overline{o^{\prime}}+\overline{o^{\prime}} ; \bar{o}\right]_{a} \|\left[o \mid o^{\prime}\right]_{b}
$$


According to the above intuitive notion of conformance, we have that $\bar{o} ; \overline{o^{\prime}}+\overline{o^{\prime}} ; \bar{o}$ is conformant to the role $a$ while $\tau ; o ; o^{\prime}+\tau ; o^{\prime} ; o$ is conformant to role $b$. But if we combine in parallel these two contracts, we obtain a wrong implementation:

$$
\left[\bar{o} ; \overline{o^{\prime}}+\overline{o^{\prime}} ; \bar{o}\right]_{a} \|\left[\tau ; o ; o^{\prime}+\tau ; o^{\prime} ; o\right]_{b}
$$

The problem here is that in the first implementation the decision about the order in which the two interactions should be executed is taken from the role $b$ while in the second implementation it is taken from $a$.

In order to avoid this problem we proceed as follows. We exploit the projection defined in Section 3 in order to extract form the choreography the "maximal" behaviour of each role. For instance, the maximal behaviours of the two roles in the above choreography are

$$
\bar{o} \mid \overline{o^{\prime}} \text { for role } a \quad \text { and } \quad o \mid o^{\prime} \text { for role } a
$$

In order to check whether a contract is conformant to a role, we then consider the refinement relation defined in Section 4, Our subcontract relation does not allow to refine $o \mid o^{\prime}$ with $\tau ; o ; o^{\prime}+\tau ; o^{\prime} ; o$ because, for instance, $[\bar{o}]_{a} \|\left[o \mid o^{\prime}\right]_{b}$ is a correct system while $[\bar{o}]_{a} \|\left[\tau ; o ; o^{\prime}+\tau ; o^{\prime} ; o\right]_{b}$ is not. For this reason, we do not consider $\tau ; o ; o^{\prime}+\tau ; o^{\prime} ; o$ conformant to the role $b$ as assumed by the first intuitive notion of conformance.

We are now ready to formally define our notion of conformance.

Definition 16 (Conformance). Given a choreography $\mathcal{I}$ with roles $a_{1}, \cdots, a_{n}$, and a role $a_{i}$ with $i \in\{1, \ldots, n\}$, we say that a contract $C$ is conformant to the role $a_{i}$ of $\mathcal{I}$, denoted $C \bowtie_{a_{i}} \mathcal{I}$, if

$$
C \preceq_{O} \operatorname{proj}\left(\mathcal{I}, a_{i}\right) \text { with } O=\bigcup_{j \in\{1, \ldots, n\}-\{i\}} O\left(\operatorname{proj}\left(\mathcal{I}, a_{j}\right)\right)
$$

Notice that we consider the refinement relation $\preceq_{O}$ parameterized on the set of output names occurring in the other "maximal" contracts. In this way, a contract could be conformant even if it adds input actions on new names that do not occur in the initial choreography.

We conclude this section proving that this notion of conformance solves our initial problem, i.e., combining in parallel contracts that are conformant to the roles in a given choreography, the obtained contract system is a correct implementation of the given choreography. We first formalize the notion of choreography implementation, after we prove this result.

Definition 17 (Choreography implementation). Given a choreography $\mathcal{I}$ and a contract system $\mathcal{S}$, we say that $\mathcal{S}$ implements $\mathcal{I}$ if $\mathcal{S} \downarrow$ and the weak complete traces of $\mathcal{S}$ are included in the weak complete traces of $\mathcal{I}$.

Theorem 4. Given a choreography $\mathcal{I}$, with roles $a_{1}, \cdots, a_{n}$, satisfying the connectedness conditions, and the contracts $C_{1}, \ldots, C_{n}$ such that $C_{i} \bowtie_{a_{i}} \mathcal{I}$ for $i \in\{1, \ldots, n\}$, we have that $\left[C_{1}\right]_{a_{1}}\|\ldots\|\left[C_{n}\right]_{a_{n}}$ implements $\mathcal{I}$. 
Proof. By definition of conformance we have that if $C_{i} \bowtie_{a_{i}} \mathcal{I}$, then $C_{i}$ is an appropriate refinement of $\operatorname{proj}\left(\mathcal{I}, a_{i}\right)$. By the Corollary 1 we have that the system $\left[\operatorname{proj}\left(\mathcal{I}, a_{1}\right)\right]_{a_{1}} \| \ldots\left[\operatorname{proj}\left(\mathcal{I}, a_{n}\right)\right]_{a_{n}}$ is an implementation of $\mathcal{I}$. By Theorem Q we can independently replace each contract $\operatorname{proj}\left(\mathcal{I}, a_{i}\right)$ with the corresponding contract $C_{i}$. In fact, the latter is in singular subcontract pre-order $\preceq$ with the former, by the theorem we know that the singular subcontract pre-order is also a "general" subcontract pre-order, and by the Definition 120 we know that refining according to the subcontract pre-order preserves correctness and does not add any additional complete trace. Thus, also the obtained refined system is an implementation of $\mathcal{I}$.

\section{Conclusion and Related Work}

We have considered the problem of checking whether a service can play a given role in a choreography. The proposed solution, obtained extending and combining previous works presented in 9 and 24, is based on the idea of verifying whether the contract of the considered service is a refinement of the "maximal" contract describing the behaviour of the considered role. This "maximal" contract can be extracted from the given choreography via projection. As a final remark, we observe that as consequence of the results we have proved in the paper, the following procedure can be used to verify the conformance of the contract $C$ with the role $a$ in the choreography $\mathcal{I}$ :

- execute the projection function in Definition 1 to obtain the contract $D=$ $\operatorname{proj}(\mathcal{I}, a)$;

- exploit the algorithm for checking should-testing pre-order defined in [12] as described in Theorem 3 to check whether $C \preceq_{O} D$ where $O$ is the set of names on which roles different from $a$ performs output actions in $\mathcal{I}$.

We now discuss the related results that we have proved in our previous papers. Detailed comparisons of our results with work from other authors can be found in the corresponding papers $9 / 1 / 3 / 5 / 4$.

In [9] we have considered different semantics for the input-output communication operations. In this work we have considered for choreographies and contract systems a synchronous, delayed semantic (delayed in the sense that interactions in choreographies and outputs in contract systems are executed in two steps, the first one being a $\tau$ action). In 9], on the contrary, the semantics of the choreography (called IOC, for interaction-oriented choreography) is not delayed, and for contract systems (called POC, for process-oriented choreography) two possibilities are considered: either a synchronous non-delayed execution, or an asynchronous one. The asynchronous one differs from the delayed one since the continuation of the output becomes enabled before the input action has been executed. Also, since in the asynchronous models there are two actions, the output and the input, corresponding to each choreography interaction, there are different possible definitions for the behavioural correspondance between choreography and contract system. For instance, the choreography may specify the 
order of inputs, of outputs, or of both. In [9] different cases are analyzed and compared, and the connectedness conditions for each of them are discussed. Also, the behavioural correspondence is modelled resorting to different variants of (weak or strong) bisimilarity.

We now summarize the results that we have obtained in [2]1|3|5|4 about contract refinement in different scenarios. Note that, with respect to the machinery reported in this paper, the work in 21131514 considers the presence of loops, thus admitting infinite behavior of contracts.

The first mean of classification of possible scenarios is based on the amount of knowledge about the (initial) behavioral description of the other roles the conformance relation may depend on. We considered: knowledge about the whole choreography (full knowledge about the behavior of other roles) or knowledge restricted to input types (receive operations) and/or output types (invoke operations) that the other roles may use. Note that in this paper we used knowledge of the latter kind. The second mean of classification of possible scenarios is based on the kind of service compliance assumed (i.e. of the principle assumed for assessing when multiple services work well together and form a correct system). We considered: "normal" compliance, as reported in this paper, where service interaction via invoke and receive primitives is based on synchronous handshake communication and both receive and invoke primitives may wait indefinitely (with no exception occurring) for a communication to happen (the standard CCS synchronization); "strong compliance", where we additionally require that, whenever a service may perform an invoke on some operation, the invoked service must be already in the receive state for that operation; "queue-based compliance", where service interaction via invoke and receive primitives is based on asynchronous communication: the receiving service puts invoke requests in an unbounded queue. Concerning service compliance we considered in all cases the fair termination property, i.e. for any finite behavioral path of the system there exists a finite path from the reached state that leads all services to successful termination. This guarantees that the system is both deadlock and, under the fairness assumption (i.e. whenever a choice is traversed infinitely often every possible outcome is chosen infinitely often), live-lock free.

Our results are summarized in the following.

- Knowledge about the whole choreography (direct conformance relation with respect to a choreography for a certain role): the maximal independent conformance relation does not exist, no matter which kind of service compliance (among those mentioned above) is considered.

- Knowledge about other initial contracts limited to input/output types they use (conformance by means of refinement of a single contract parameterized on the I/O knowledge about the others, as in this paper):

- In the case of "normal compliance" we have that: for unconstrained contracts the maximal independent conformance relation does not exist; for contracts where outputs are internally chosen, as in the case of this paper, (more precisely for contracts such that the output persistence property holds) the maximal independent conformance relation 
exists and knowledge about input types is irrelevant; for output persistent contracts where locations expressing a unique address for every system contract are introduced and outputs are directed to a location, the maximal relation exists and knowledge about both input and output types is irrelevant.

- In the case of "strong compliance" we have that: for unconstrained contracts (where outputs are directed to a location identifying a unique system contract) the maximal relation exists and knowledge about both input and output types is irrelevant.

- In the case of "queue-based compliance" we have that: for unconstrained contracts (where outputs are directed to a location identifying a unique system contract) the maximal relation exists and knowledge about both input and output types is irrelevant.

For every maximal refinement relation above (apart from the queue-based one), we provide a sound characterization that is decidable, by resorting to an encoding into should-testing [12, a fair version of must testing. As a consequence we obtain:

- An algorithm (based on that in 12]) to check refinement.

- A classification of the maximal refinement relations with respect to existing pre-orders as, e.g., (half) bisimulation, (fair/must) testing, trace inclusion. In particular we show that the maximal refinement relations are coarser with respect to bisimulation and must testing preorders (up to some adequate encoding and treatment of fairness) in that, e.g., they allow external nondeterminism on inputs to be added in refinements.

Acknowledgement. We thank Claudio Guidi and Fabrizio Montesi co-authors of the paper 9] (which Section 3 of this paper is based on). We also thank the members of the SENSORIA project for numerous insightful discussions about choreography and contract languages.

\section{References}

1. Bravetti, M., Zavattaro, G.: Towards a unifying theory for choreography conformance and contract compliance. In: Lumpe, M., Vanderperren, W. (eds.) SC 2007. LNCS, vol. 4829, pp. 34-50. Springer, Heidelberg (2007)

2. Bravetti, M., Zavattaro, G.: Contract based multi-party service composition. In: Arbab, F., Sirjani, M. (eds.) FSEN 2007. LNCS, vol. 4767, pp. 207-222. Springer, Heidelberg (2007)

3. Bravetti, M., Zavattaro, G.: A theory for strong service compliance. In: Murphy, A.L., Vitek, J. (eds.) COORDINATION 2007. LNCS, vol. 4467, pp. 96-112. Springer, Heidelberg (2007)

4. Bravetti, M., Zavattaro, G.: A Foundational Theory of Contracts for Multi-party Service Composition. Fundamenta Informaticae 89(4), 451-478 (2008)

5. Bravetti, M., Zavattaro, G.: Contract Compliance and Choreography Conformance in the Presence of Message Queues. In: WS-FM 2008. LNCS. Springer, Heidelberg (2008) 
6. European Integrated project SENSORIA. Web site, http://www . sensoria-ist.eu

7. Fournet, C., Hoare, S.T., Rajamani, S.K., Rehof, J.: Stuck-Free Conformance. In: Alur, R., Peled, D.A. (eds.) CAV 2004. LNCS, vol. 3114, pp. 242-254. Springer, Heidelberg (2004)

8. Hoare, C.A.R.: Communicating Sequential Processes. Prentice-Hall, Englewood Cliffs (1985)

9. Lanese, I., Guidi, C., Montesi, F., Zavattaro, G.: Bridging the gap between Interaction- and Process-Oriented Choreographies. In: SEFM 2008. IEEE Computer Society Press, Los Alamitos (2008)

10. Milner, R.: Communication and Concurrency. Prentice-Hall, Englewood Cliffs (1989)

11. OASIS. Web Services Business Process Execution Language Version 2.0 OASIS Standard, http://docs.oasis-open.org/wsbpel/2.0/0S/wsbpel-v2.0-0S.pdf

12. Rensink, A., Vogler, W.: Fair testing. Information and Computation 205(2), 125198 (2007)

13. World Wide Web Consortium. Web Services Choreography Description Language Version 1.0, http://www.w3.org/TR/ws-cdl-10/ 